East African Medical Journal Vol. 85 No. 3 March 2009

INFORMED CONSENT FOR CLINICAL TRIALS: A REVIEW

V.M. Lema, MBChB, MMed (Obs. \& Gynae) Regional Medical Director, M. Mbondo, DrPH, CHES, Senior Technical Officer, Surveillance and E.M. Kamau, PhD, Research Associate II, Family Health International, Africa Regional Office, P.O. Box 38835 - 00623, Nairobi, Kenya

Request for reprints to: Prof. V. M. Lema, Family Health International, Africa Regional Office, P.O. Box 38835 - 00623 , Nairobi, Kenya

\title{
INFORMED CONSENT FOR CLINICAL TRIALS: A REVIEW
}

\author{
V.M. LEMA, M. MBONDO and E.M. KAMAU
}

\begin{abstract}
Objective: To identify and highlight challenges related to informed consent process for clinical trials in sub-Saharan Africa.

Data sources: Published original research findings and reviews in the English literature, together with anecdotal information from our current professional experiences with clinical trials.

Design: Review of peer-reviewed articles.

Data extraction: Online searches were done and requests for reprints from corresponding authors and institutional subscription.

Data synthesis: Information categorised accordingly.

Results: Informed consent for clinical trials conducted in sub-Saharan Africa (SSA) is not always "truly informed" or "truly voluntary". Guidelines for obtaining informed consent are often difficult to implement because of low literacy levels, socio-economic and cultural factors. The local ethics committees, whose role is critical in informed consent, are weak, ill-equipped or non-existent in some countries. Many participants may have incomplete understanding of the various aspects of the clinical trials due to language barriers, the way information is disclosed or terms used for informed consent documents. In some settings, clinical trials are the only access to health care services for the local population. Further, participants may enroll with perceived notion of cure of their conditions, for monetary or material benefits.

Conclusions: There is need for national guidelines on clinical research including ethics review, compensation of subjects, requirements for research investigators, facilities and ethics committees as well as budgetary allocation. These guidelines must not only address specific and unique local circumstances but also meet minimum international clinical research standards. Local bioethics and research capacity should be developed and strengthened with research sponsors contributing towards this. Local research is needed on the validity and reliability of informed consent for clinical trials and factors influencing that in different socio-cultural settings in SSA.
\end{abstract}

\section{INTRODUCTION}

Clinical trials are used to determine the efficacy and safety of new pharmaceutical products or treatment procedures. Randomised and controlled clinical trials (RCTs) are considered the safest and fastest means of identifying treatments that work in human beings. The goal of a clinical trial is to answer specific questions about drugs, vaccines, new devices or therapies or therapeutic approaches of known treatments (1).

Clinical trials are becoming increasingly necessary because of emergence of new diseases afflicting mankind such as HIV / AIDS; resistance to standard drugs e.g. antimalarials and antibiotics; the need to improve the quality and prolong the lives of individuals with chronic and debilitating conditions such as cancer, diabetes and hypertension and congenital anomalies/birth defects. There is also an increasing demand from the public due to heightened awareness, for prevention and treatment of common and debilitating conditions. Equally important are social and economic concerns for communicable and chronic diseases.

Ideas about clinical trials may be originated by scientists including health professionals based on their clinical and public health experiences, the pharmaceutical industries that wish to market their products, internationalorganisations, orgovernmental agencies such as regulatory authorities. 
As clinical trials are costly and resource-intensive and take long to realise their goals, it is imperative that the results thereof are not only credible but also reliable. They must not only show that the drug works and is safe for human beings but identify the ideal conditions for its use as well as potential adverse effects (1-4). They need to be conducted in diverse settings and communities, especially those most affected by the condition under study. However due to cost constraints, representative countries and localities are selected to represent the wider community for which it will be used. It is assumed that the data from these can be pooled together and analysed and the results thereof generalised $(5,6)$. To this end internationally acceptable standards for conducting clinical trials must be adhered to at all stages of a study.

Good clinical practice (GCP) is an international ethical and scientific quality standard for the design, conduct, performance, monitoring, auditing, recording, analysis and reporting of clinical trials, that provides assurance that the data and reported results are credible and accurate and that the rights and integrity of trial subjects are protected (6). Study subjects should only include eligible participants, who are identified through the screening and consenting process, an essential aspect for a successful clinical trial. The individual's consent must be truly informed and truly voluntary $(7,8)$.

There are concerns whether informed consent by study participants especially in the developing world are genuinely informed and voluntary (9-11). There is very limited literature on informed consent and its role in clinical trials in sub-Saharan Africa. With internationalisation of clinical trials and the majority of specific types such as those on HIV-prevention taking place in the region, the concerns on informed consent are even more critical.

Clinical trials use relatively complex and detailed patient information and informed consentdocuments, which are written in academic language and that of the sponsor's country of origin. Although these are translated into national or local languages of the host countries, individuals who are educationally disadvantaged as is the case in most of SSA rural areas may have problems comprehending the disclosures (9-13). The fact that a majority of the clinical trials take place within public health facilities, participants may not be able to discern between routine care and research (14). Cognisant of the special situations of developing countries, the CIOMSissued guidelines on how these ethical principles should guide biomedical research involving human subjects (5).

Making a decision to participate in a clinical trial involves understanding of the potential risks and benefits as well as ones rights and responsibilities. This is said to have cognitive, emotional, motivational and value-based components (10). It has been argued that the decision to participate is often made before and independent of the disclosed technical information during counselling and consenting process (15), and that personal and cultural values play a major role in this.

This article highlights challenges related to informed consent from our experiences in participating in various clinical trials in the SSA region. It proposes strategies to address some of the shortcomings.

\section{INFORMED CONSENT}

Definition: Informed consent is an autonomous authorisation by an individual for involvement in a research study or to receive medical care and the documentation thereof (15). It is fundamental to the ethical conduct of a clinical trial and a critical component of the process thereof. Through the informed consent process, the potential study subjects learn about the important facts related to the clinical trial which enable them to make informed decision to participate or not. It is essential for protecting the rights and ensuring the safety and privacy of potential study participants. The Belmont Report (1979) defines informed consent as essential to the principle of respect (3).

Origins: The origin of informed consent is credited to the Nuremberg Code (1947), which made voluntary consent a requirement in clinical research studies (8). The Code together with the Declaration of Helsinki (1978) which focused on the obligations of the investigators to research subjects (4) are considered the mainstay of ethical guidelines for research involving human subjects. Informed consent is now enshrined in the WHO International Ethical Guidelines for Biomedical Research Involving Human Subjects (1993) (5).

\section{Principles:}

Table 1

Key components of informed consent

- Full disclosure - on the purpose of the study, its duration, the related risks and benefits and alternative therapeutic options

- Information - should be in writing in a form that is easily comprehensible and the subject must understand fully the information given

- The consent - must be obtained freely and the subject should be free to refuse or withdraw at any time without prejudice

- The subject - must be competent to give consent.

Informed consent is an ethical principle of autonomy and self-determination (16). The ethics 
committee of the sponsoring country and that of host country must review the research protocols for among other things protection of subjects' rights, safety and well-being. The research team should be competent and knowledgeable on interpersonal communication. The interaction between investigators and subjects must be continuous throughout the period of the study and beyond $(6,15)$.

Protection of trial subjects is the primary concern of all parties involved in the conduct of a clinical trial and review of the protocol. The principle of informed consent requires that health professionals or research investigators explain to the patients or subjects what is involved and ensures one understands before consenting. This must be done prior to any procedures (4-6).

The doctrine of informed consent dictates that research participants should enter into a research study voluntarily and with adequate information. Informed consent is given by a competent individual who has received the necessary information, who has adequately understood theinformation and who after considering the information has arrived at a decision without having been subjected to coercion, undue influence, inducement or intimidation. It is premised on the notion that individuals have the right and the ability to make decisions in their own interest and to act upon them (17). It protects the individuals freedom of choice and respects the individual's autonomy.

Challenges:

Table 2

Constraints in the application of ethical principles in less-developed countries (LDC's)

\begin{tabular}{ll}
\hline Ethical guideline & Constraint to application in LDC's \\
\hline $\begin{array}{l}\text { Review of research protocols by ethics committee/ } \\
\text { institutional review boards }\end{array}$ & Lack of experienced, independent EC/IRB's
\end{tabular}

Reasonable risk-benefit ratio

Assessment of risk-benefit ratio may be biased by prevalence of related clinical conditions

Adequate plans for the care and compensation of participants for injuries directly related to the research

Individual informed-consent from all participants

Equal regard for all participants

Equitable distribution of the burden and benefits of research
Participants may not be aware they are entitled to compensation

Participants may not report adverse events or injuries

Low literacy levels, social, economic and cultural constraints may prevent informed independent consent

Cultural or political restrictions to participation of members of certain social groups, or based on gender

Incentives may draw participants disproportionately from the poorest segments of the population

The individuals may not afford the drugs when approved and registered due to cost

Bias towards more urban or peri-urban areas for ease of access by research team 
i) Ethics Committees and Institutional Review Boards: Approval of research protocol by an ethics committee or institutional review board (EC/IRB) is a requirement for any clinical trial whether international or local (4-6).

These are independent bodies consisting of health care professionals and non-medical members, whose responsibility is to verify that the human rights, safety, integrity and well-being of human subjects participating in medical research are protected and to consider the general ethics of the trial thereby providing public assurance of that protection $(4,6)$. They are supposed to review the research protocol, the informed consent documents, suitability of investigators involved in the trial and adequacy of the facilities to be used for the various aspects of the trial. They should also receive and appraise progress reports from the investigators as well as any new information related to the study from the investigators as it may arise. One of the main functions of the EC/IRB is to ensure that potential study subjects are adequately informed about the clinical trial, the benefits and risks thereof, their rights to withdrawal at any time and alternative treatment options $(18,19)$.

There have been concerns about the capacity for ethics review of clinical trials. It is acknowledged that this capacity is not universal and the developing countries such as those in SSA have major limitations in this regard (19-21). Sometimes institutional review boards from developed sponsor countries review protocols that will be conducted in one or more host developing countries. Many of the members on these boards may not be familiar with the host country's culture, language, social and ethical norms, level of health care and other factors essential for a thorough ethical review of the protocols. In majority if not all international clinical trials, the EC of the country of origin and the host have to review and approve the protocols. In some cases there are several bodies within the country that have to review a protocol. Unless the requisites are harmonized, there may be conflicting decisions. This is more critical in multicountry or multi-center trials.

There has been limited research examining procedures, strengths and challenges facing ethics committees in developing countries. Many EC/IRBs especially in SSA have difficulties in recruiting and retaining community members who have sufficient time, interest and education to comprehend the complex scientific protocols they review. They may therefore approve trials which may expose participants to undue harm. Secondly they may not be able to critically review the informed consent documents and procedures to ensure safety of the participants. From the experience of one of us who had the privilege of serving on an institutional review board in one country in SSA for a period of six years, it is difficult to get capable non-medical members as required to serve on ECs, and those who agree often miss committee meetings for one reason or another and when they participate their contribution is often insignificant.

Some members of IRBs demand or expect to be remunerated for their time and effort. If they don't get paid their participation may be compromised. There are no guidelines as to whether they should be paid and how much. Secondly if and when they get paid as it happened in the committee referred to above, what may be considered adequate by one may be inadequate or too much for another which may exert an "undue inducement" on his/her level of commitment.

There may be conflict of interestif a member of the EC/IRB has financial connection with the sponsoring organisation, or the manufacturer, or who may gain financially from the results of the study. He/she may approve a protocol without considering the rights of the subjects. Others may influence enrollment of subjects who are relatives or even themselves into the clinical trials.

ii) The research team: The composition and quality of the research team is one of the key determinants for a successful clinical trial. In most SSA countries the principal investigator (PI) who is the overall in charge of the clinical trial is a physician, specialist in the discipline under which the study is being conducted. The day to day management of the study including screening, counselling and consenting of potential study subjects is handled by either clinical officers or nurses. These cadres have little or no training on research methodology and ethics and may have limited knowledge of the health condition under study. They may therefore not be able to provide adequate information especially in response to subjects' questions, thus compromising their informed consent. Some research investigators are foreigners and may not know the social, cultural or economic context of the host countries and may not have personal interest in the health care of the local community. The principal investigator is responsible for ensuring that information in the consent form and other related documents is accurately explained to and apparently understood by the subjects. However there are no specific guidance or standards for assessing this "explanation" and "understanding".

Secondly, if their remuneration is pegged on the number of eligible participants recruited, which is the case in most studies, they may take short cuts and not provide full disclosure especially on risks involved or available alternative treatment options. They may stress on the benefits of the study, thus coercing the subjects to enroll in the study in order to realise the numbers $(22,23)$.

Most of the clinical trials in SSA are conducted within public health facilities. Members of the research team are usually staff working in the same facilities and therefore well known to the potential subjects or members of their families. The subjects may therefore consent because of "trust" in the 
health team (24), a feeling that they are expected to participate by the health team (25), or for fear that if they do not participate they may experience problems later on when they seek health care at the same facility (26). In a study by Karim et al (11) in South Africa, $88 \%$ of the participants felt that they were compelled to participate in the clinical trial while $28 \%$ thought if they refused to participate it may compromise their care.

The physician may have conflict of interest as they may have difficulty distinguishing between their primary goal of treating patients and that of a researcher i.e. to test scientific hypothesis (26). They may also want their patients enrolled into the study and influence their participation. This may influence who gets enrolled. Likewise, physicians with shares or other financial interests in the study results may have conflict of interest and this may impact negatively on the informed consent process as they will put their personal gains before subjects' safety (27). A researcher in an academicinstitution may have conflict of interest because he/she has to discern what is good for the current patient, future patients i.e. the good of society, the institution and his / her career development (25). The research team may withhold key information or not follow good clinical practice guidelines if there is no proper oversight by ethics committee.

iii) Comprehensionandretentionofdisclosed information: Consent for clinical trials is considered "informed" when the subjects giving it understand the purpose and nature of the study, what is expected of them, and the potential benefits and risks resulting from the study (4-6). There are concerns whether clinical trial subjects do really understand enough about the study before consenting. A number of studies in industrialised countries have shown the existence of gaps in the information provided and the understanding by study subjects (9-13). That being the case, it is not far-fetched to postulate that the scenario is even worse in SSA, cognisant of the high poverty and low literacy levels and poor access to quality health care services, which make them vulnerable to exploitation for research purposes. From our experience this is a fairly common phenomenon.

Clinical trials conducted in SSA originate from developed countries and usually utilise relatively complex and detailed patient information documents written in academic language, sometimes imported from the country of the study origin. Although IRBs insist that these be translated into locally understood or spoken languages, some words do not have their equivalence in local languages. Where the IRB does not insist on translation into a local language, the researchers may administer the informed consent in a foreign language. In a study in one country in Africa where less than $25 \%$ of the population understand or speak English we were shocked to note that the research team was disclosing the information and consent process in English and only asking the subjects to append their signatures or thumb prints to the consent forms which were also in English. As a result study subjects who are educationally challenged may have problems comprehending the disclosed information. The length and complexity of the informed consent documents and related subject information may impact negatively on a subject's understanding of the clinical trial related information (29). The way the information is packaged and presented such as presenting risks or benefits in percentages, may not be well appreciated especially by the educationally disadvantaged individuals.

Subjects consent to participating in clinical trials is a basic ethical and legal pre-condition to conscientious medical practice and the ethical principal of respect for person's autonomy requires that they understand the accessible treatment options among which they may choose (30). The decision to do either can only be genuine and valid if it is based on adequate information. The major challenge is how much information should be disclosed to be considered "enough" for appropriate decision, but at the same time not "too much" to frighten subjects either from participating or into compliance!

iii) Therapeutic misconception: At times study subjects fail to appreciate the distinction between the imperatives of clinical trials and those of routine medical care, thus attributing therapeutic intent to research procedures. This is referred to "therapeutic misconception" (31). The subjects may not appreciate the implications of their decisions. They may expect personalised care, which is part of routine medical care but not necessarily part of clinical trials.

Therapeutic misconception is a serious problem for the informed consent process in clinical trials. It may arise because of patients' faith in the health care providers and belief that they will do nothing that is not in their best interest, or may be as a result of the disclosed information by the research team which may reinforce a subject's predilection for, or misinterpretation of the information so disclosed (14). Benson et al (32) observed that some researchers may underscore the therapeutic potential of the studies, thus influencing potential subjects' decision to participate.

Subjects who are also patients who do not adequately appreciate the purpose and methods of research studies are ill-equipped to evaluate related benefits and risks and fail to recognise how personal care may be compromised by research procedures, such as randomisation and use of placebos (14). In a study by Applebaum et al (33) involving subjects of four psychiatric clinical trials in the US, $40 \%$ stated that they had been assigned to treatment arm based on their therapeutic needs and $44 \%$ did not recognise the use of placebo. Krosin et al (9) in their study in Mali reported that $74 \%$ of the participants did not understand that they were enrolled in an investigation and not receiving therapy. Many research findings suggest that personal benefits is the major motive for participating in clinical trials even when they hold 
little prospect of benefit $(34,35)$. When the expected results are not realised the backlash can be serious leading to withdrawal from the study or resentment and anger towards the research team.

The disease condition and its perceived or known prognosis such as cancer and HIV / AIDS may impact on study subject's therapeutic misconception. Therapeutic misconception is considered to be widely spread globally and fairly common in clinical trials (14). However, it has greater implications on the informed consent process in clinical trials conducted within SSA such as those on HIV / AIDS, which are currently quite common, as participants may enroll expecting to get full protection or cure of the infection, as we have witnessed in some of the HIV prevention trials in the region.

iv) Disease condition and severity and its impact on informed consent: Each clinical trial focuses on a specific health condition. It may be aimed at testing a preventive strategy such as a vaccine or prophylactic treatment or comparing the efficacy and safety of a new drug or drug combinations or treatment approach to a standard one or placebo. Study subjects in preventive studies are healthy individuals whereas those in treatment trials are patients with varying degrees of severity of the condition.

It is postulated that a subject with an immediate life-threatening disease and notherapeutic alternative might retain less from investigators' disclosure than would a subject with less severe disease or healthy volunteers. Likewise patients with lifethreatening conditions are less autonomous than healthy volunteers in their decision-making. Their motivation for participating in the trial is cure of their health condition and may therefore consent without considering the risks involved (34).

Schaeffer et al (34) in their study in the US found that healthy volunteers retained information the best while severely ill patients retained the least. Likewise the sick reported that the informed consent document did not have an effect on their decision to participate in the trial. Individuals with serious health conditions like cancer and HIV/AIDS may enroll in clinical trials with goals that are at variance with the goals of the research protocols. The consent document is rated as less useful or relevant by study subjects with advanced disease conditions. They have poor retention of disclosed information on risks perhaps due to denial of unpleasant realities, or they want to avoid disturbing thoughts associated with risk information.

It is not clear whether the nature of the disease has an influence on the informed consent process. However cognizant of the foregoing, it is very likely that conditions like cancer and HIV/AIDS have similar impact as severe illness. That being the case, and considering the fact that majority of HIV / AIDS related clinical trials are being conducted in SSA because of the high prevalence and incidence, this may have great impact on the subjects consent for enrollment into the studies. They may be too eager to append their signatures on the informed consent forms without really understanding what they are consenting to. The HIV / AIDS epidemic has raised ethical concerns not previously envisaged. In some instances peopleliving with HIV / AIDShaveclaimed a right to access to clinical research with the perceived notion of cure! When participants in a trial one of us was involved in were asked why they enrolled, one of the main reasons was that they expected full protection against HIV infection.

v) Participants' remuneration: Renumerating research study subjects is a thorny research ethics issue. Most international and national guidelines on research ethics warn against inducement of subjects to participate, but do not explicitly say what should be done. The CIOMS (1993) provide the most comprehensive guidelines on this issue. While discouraging undue inducement of subjects, they stipulate that subjects may be paid for inconvenience and time spent and be reimbursed expenses incurred in connection with their participation in the research. They may also receive free medical services (5). There are different views though regarding remuneration of study subjects (36). Some regard participation as a social responsibility deserving no payment but recognition for the time and efforts of participants (36). Others recommend wage payment based on hourly rate for unskilled workers (37). A few ethics committees, such as that of South Africa, have specified the actual amount to be paid to subjects in clinical trials (11). While some participants consider this appropriate, others feel it is too much or too little $(38,39)$.

In a number of clinical trials in SSA especially those involving children on HIV / AIDS or malaria in pregnancy, the subjects get other material benefits such as supplementary/complimentary feeds, mosquito nets, baby clothes, free treatment of other health problems. Likewise the study subjects get better care during the trial which they would not get in routine medical care. Their hospital bills e.g. for accommodation, laboratory tests and drugs are paid for by the research. Whereas these are not direct payments to study subjects they are regarded as such and may also motivate a family or an individual to participate in a study.

Payment of study subjects is contentious especially in the context of developing countries. Money means different things to different people. It is not clear though how much money constitutes undue inducement (39). The Belmont Report states that undue influence occurs through an offer of an excessive unwanted inappropriate or improper reward or other overtures in order to obtain compliance (3). Such overtures may compromise the informed consent process and scientific validity of a clinical trial (36-39). Whereas the CIOMS guidelines state that the study subjects may also receive free medical services (5), it does not specify whether these 
are related to the health condition understudy or any! The line between "fair compensation" and "undue inducement" is often difficult to determine.

Whereas it is necessary to pay study participants for their inconvenience and time spent and effort, as well as reimburse expenses such as transport costs, financial compensation is an important reason for many people's participation in clinical trials. This is particularly so in SSA where majority of potential study subjects are poor and have no definite means of financial sources. Monetary compensation and gifts such as those mentioned above may exert undue inducement to participate. This will undoubtedly compromise their decision-making process or willingness to explore the risks and benefits of the study before enrolling.

vi) Social and cultural factors: Majority of clinical trial protocols are written in either English or French depending on the originator and/or sponsor as well as the host country in which the study will be conducted. The informed consent documents as well as related information sheets are translated into the host country's national language and then in some cases to the local community language or dialect. The main concern in this process is the fact that some dialects such as the Bambara in West Africa do not have written form. Secondly some words donot have direct translation in local languages or dialects. Good examples are randomisation, placebo, and clinical trial. In translating these words the meaning may be distorted. While trying to explain what one word in a foreign language means in a local language it may require a whole sentence of paragraph, making the document too long and not user-friendly. Thirdly, due to the low literacy levels in SSA especially among the rural majority, who must be included in clinical trials conducted within the countries for them to be truly representative, the potential subjects may notbe able to read and understand what they are consenting to. For the educationally disadvantaged the researchers may read out for them and just ask them to append their signatures or thumb-prints as a sign that they have understood what the study entails and their rights as we observed in one trial. In such cases the consent cannot be said to be truly informed and/or truly voluntary!.

The same is true regarding poor patients seeking medical attention who are categorised as vulnerable to exploitation by researchers (12). Due to their social status, they may not ask too many questions or may relinquish their autonomy to the research team on the assumption that they know what is in their best interest. By signing the consent form, some participants may feel that it is a binding contract and therefore contradict the concept of voluntarism and right to withdraw even when they may have side effects. Due to previous experiences with signing documents, some participants may decide not to participate.
Theissue of culture and informed consent process especially for the developing countries has been the subject of recent scientific research and publications $(40,41)$. There are concerns regarding consenting for minors, adolescents and female subjects in particular. We have in the course of our participation in clinical trials in various parts of SSA, seen cases where a mother who is a legal guardian of a child consents for her child to be enrolled in a clinical trial, only for the father to withdraw the consent and rebuke her and the research team because he was not consulted, or parents-in-law of the woman in patrilineal or woman's parents, brother or uncle in matrilineal societies may have to be consulted to give their nod before one can consent. It becomes confusing in such situations as to who really is supposed to give consent for a child to be enrolled in a children's clinical trial. It is also not clear who should give consent in cases of adolescent females, even when already married and / or are mothers themselves. Can she be regarded as an adult by virtue of her marital status or is she still a minor legally? What is more disturbing is that the same adolescent mother is a legal guardian for her child and can give consent for medical care! The law and research ethics are silent on this issue!

In most societies in Africa a married woman needs her husband's endorsement or approval to receive certain types of health care services such as contraception and surgery. For clinical trials on reproductive issues e.g. vaginal microbicides, condoms, with sexual behaviour as one of the requisites for assessing success or failure of the study, cooperation of the two individuals is essential. The same can be said of trials which require sexual abstinenceor whichmay impact of sexual performance or enjoyment thereof such as male circumcision or condom use. In such cases whose consent is required; both or the subject's and who is the subject in this case? Whose autonomy are we talking about in this case? What happens when the spouse is not consulted in advance? In one study involving vaginal gel, which made the vagina wetter than expected in a society which prefers dry sex, a number of the participants had to withdraw as their husbands threatened them with divorce and some were abandoned or beaten. In yet another study testing acceptability and use of the female condom in reducing STI's the study had to be abandoned because of refusal by the male partners of the subjects.

It is argued that local community opinion on pertinent beliefs and practices should inform ethical decision-making in health research. In some cultures a woman can not be alone with a man even in the health care facility. Another woman such as one's mother, sister or mother-in-law must be with her constantly. In a study one of us was involved in, which had pregnancy as an exclusion criterion, required asking potential subjects about menstrual history, their sexual behaviour and contraceptive use, and when in doubt to do a pregnancy test. There was strong 
resistance from the community as it was considered taboo to ask girls below certain age about sex and do pregnancy tests. There are problems in such situations during the consenting process since there will not be any confidentiality. Health care providers will have a difficult task when asking relevant but sensitive questions or when disclosing results to the subject. The subjects may hold back vital information on questions which would influence their eligibility for the study. Dickens et al (30) compare patient-physician relationship in disclosure of secrets which they would not even disclose to their spouses, or close relatives, to that of a congregant-priest in religious confession.

The informed consent as it is understood derives mainly from the western notion of individuality. It refers to the individual rights and autonomy. However in some countries in the developing world there is more of communalism wheretheindividual considers himself/ herself as part of the larger society and whatever s/ he does is for the good of the society (26). The procedures of disclosure and consent norms as envisaged in the western model may not work. In some instances the community through its leaders has to give a nod for its members to be involved in any clinical trial as it is seen to infringe on its cultural values as we have experienced in some of the trials wehave been involved. The members can only consent to participate in clinical trials with approval of the community elders. When this happens we wonder whose rights are being protected the individual's or that of the community? There are also issues with definition of the "community" in such cases. Moodley (26) avers that concepts such as riskbenefit ratio and fair treatment of trial participants may be interpreted differently in traditional rural African communities. It has been argued that in some cultural contexts it may be appropriate to obtain agreement from a particular community or assent from a senior family member, before any prospective participant in research is approached. In either case the individual's consent must always be obtained

\section{DISCUSSION}

Informed consent is a critical component of clinical trial procedures. It is a continuous and interactive process that enables an individual to voluntarily decide whether to participate in a research study or not as well allows one to withdraw at any time. It is meant to ensure and protect the individual's autonomy, dignity and rights. Truly informed and voluntary consent is necessary for the results of a clinical trial to be credible and reliable. The subjects must therefore receive sufficient and correct information on the study itself and the consent process. There should be effective communication in an ongoing manner throughout the study period to ensure high level of comprehension and retention of disclosures $(2,4)$.

Studies in the region and elsewhere have shown that there are major challenges in achieving truly informed and voluntary consent for clinical trials $(7,9$
- 13, 25, 36, 42 ). Whereas these challenges are global, they are more pertinent in SSA and other parts of the developing world which are increasingly seeing more clinical trials especially with the HIV / AIDS pandemic, with its weak health care delivery systems and administrative capabilities as well as low literacy and high poverty levels.

The ethics committees and institutional review boards in some countries in Africa which are necessary toensure protection of the safety, rights and well-being of study subjects, are weak, ill-equipped or nonexistent in some cases. They often approve protocols without even reviewing them or understanding the contents therein, because of limited knowledge of research and scientific terms (19-21).

As majority of clinical trials conducted in SSA originate and are sponsored by manufacturers or organisations based in industrialised countries, the members of the research team who are often poorly paid employees of publicinstitutions by international standards, may be compromised to carry out trials for their own benefits disregarding the rights and safety of the participants. This must be guarded against at all cost. Likewise the main researchers i.e. the principal investigators may be foreigners who have little or no knowledge of the host country's social, cultural and economic contexts and have no personal interest in the health care of the study population. They may disregard important ethical considerations which may infringe on the rights and safety of the subjects. As stated by Doyal (43) "when human autonomy and dignity are at stake, the cost of scientific progress is too high".

Comprehension of disclosed information is essential for informed decision making. Tobias et al (29) believe that informed consent can some times be needlessly cruel by the contents therein and its length. Montgomery etal (44) state that the disclosures should use tactful and through a sympathetic dialogue so that potential subjects understand the difficult scientific or medical terminologies. For the educationally disadvantaged rural and peri-urban residents of SSA this is a major challenge. The research team needs to not only be aware of that but also ensure that each participant understands the requisite information through probing questions and repetition of the disclosures and responding to subjects questions in a sympathetic manner throughout the period of study.

Whereas therapeutic misconception is a global phenomenon, it is more acute in SSA due to its uniqueness. The clinical investigators need to be aware of this possibility and ensure they minimise it as much as possible, by providing requisite information in such a way that allows subjects to make the distinction between research and routine medical care. They should also ask probing questions regarding the reasons for participating.

Whereas appropriate compensation of participants for their efforts and time as well as 
reimbursement of expenses related to the study is acceptable, there are no guidelines on what is appropriate and acceptable level $(36,38,39)$. Secondly what may be appropriate or adequate in one place and for one trial may either be too much or too little for another. Other materials given either as gifts or part of the study such as infant feeds, mosquito nets, baby clothes, may be considered an "undue inducements" especially for poor communities. There is therefore need for national guidelines taking cognisance of local situations.

Although there is no consensus on how clinical trial protocols and informed consent should be treated in different social and cultural contexts, it is wise for originators of international clinical trials to be aware of local social and cultural norms and values when designing trials for developing countries to avoid conflict with local communities as we have witnessed in some of the trials we have been involved in. At times it is advantageous to work with rather than through local communities!

Subjects' consent to treatment is basic ethical and legal pre-condition to conscientious medical practice and the ethical principal of respect for person's autonomy requires that they understand the available treatment options among which they may choose. In clinical trials the subjects have options to participate or not as well as to drop out at any time. The decision to do either can only be genuine and valid if it is based on adequate information. The major challenge is how much information should be disclosed to be considered "enough" for appropriate decision, but at the same time not so much as to coerce or frighten subjects either from participating or into compliance.

The mere formulation and publication of ethical guidelines for biomedical research involving human subjects is notenough to ensure protection and safety of participants in clinical trials. They do however draw the attention of investigators, sponsors and ethical review committees to the need to consider carefully the ethical implications of research protocols and conduct of research and the need to ensure high scientific and ethical standards of research.

\section{RECOMMENDATIONS}

Well-conducted randomised clinical trials form the most influential basis of today's evidencebased medical practice. Cognisant of increasing internationalisation of clinical trials and that for some the developing world will be its best study sites, and in order for the results thereof to be of comparable international standards, there is need to ensure that the internationally accepted good clinical practice is upheld. Informed consent must be truly informed and truly voluntary. To help achieve that we recommend that: (i) There is need for national guidelines on research including ethics review, compensation of subjects, requirements for research investigators and facilities and Ethics Committees/IRB's, as well as budgetary allocation.

(ii) Local bioethics capacity should be developed and strengthened. The EC and IRB should have appropriate training, funding and support. Organisations funding and conducting clinical trials and other research studies in SSA should contribute to the national research budgets and the funds may be used for this. At the same time each organisation could budget for training of EC/IRB which could be organised prior tostudies or on a regular basis. In particular, non-medical members of the ethics committees need to be given adequate training on their responsibilities and their value within the committee.

(iii) The pre-service curricula of health care professionals such as doctors, nurses, clinical officers etc, should include training on research ethics and methodology as well as interpersonal communication. Before starting a research study the research team should undergo training and certification on good clinical practice as a matter of routine.

(iv) The research team should dedicate one of its member or a neutral educator to spend time talking one-on-one with study subjects to ensure they understand the requisite information.

(v) Evaluation of informed consent process and procedures should be an integral part of every clinical trial so as to identify issues that need to be addressed. This should be included in the protocol and budgeted for.

(vi) There is need for more regionally and nationally relevant research studies on various aspects of the informed consent which will provide vital information to help the process in future trials.

\section{REFERENCES}

1. Fisher B. Clinical trials and values limitations. Cancer J. Clin. 1978; 28: 241-246.

2. Protection of Human Subjects, 45 CFR $\$ 46$. Available at: http://ohrp.osophs.dhhs.gov/humansubjects/guidance/ 45cfr46.htm. Accessed April 11, 2004.

3. TheBelmont Report. Ethical Principles and Guidelines for the Protection of Human Subjects of Research. Available at: http://ohrp.osophs.dhhs.gov/humansubjects guidance/belmont.htm. Accessed June 28, 2004.

4. World Medical Association Declaration of Helsinki. Ethical Principles for Medical Research Involving Human Subjects. Available at: http://www.wma.net/e/ policy/b3.htm. Accessed June 28, 2004.

5. CIOMS. International Ethical Guidelines for Biomedical research Involving Human Subjects. Geneva CIOMS, 1993. 
6. WHO. Guidelines for GCP for trials on pharmaceutical products. WHOTechnical Report Series no. 850, 1995. WHO Geneva.

7. Joubert, G., Steinberg, H., van der Ryst, E. and Chikobvu, P. Consent for Participation in the Bloemfontein vitamin a trial: How informed and voluntary? Am. J. Public Health. 2003; 93: 582-584.

8. The Nuremberg Code. Available at: http://ohsr.od.nih. gov/guidelines/nuremberg. html. Accessed June, 2004

9. Krosin, M.T., Klitzman, R., Levin, B., et al. Problems in comprehension of informed consent in rural and peri-urban Mali, West Africa. Clin. Trials. 2006; 3: 306-313.

10. Lindegger, G. and Richter, L. M., HIV vaccine trials: critical issues in informed consent. S. Afr. J. Sci. 2000; 96: 313-317.

11. Karim, Q.A., Karim, S.S. Coovadia, H.M. and Susser M. Informed consent for HIV testing in a South African Hospital: Is it truly informed and truly voluntary? A. J. Pub. Health. 1998; 88; 637-640.

12. Oduro, A.R., Aborigo, R.A., Amugsi, D., et al. Understanding and retention of the informed consent process among parents in rural Northern Ghana. BMC Medical Ethics 2008; 9:12 doi:10.1186/14726939-9-12

13. Fitzgerald, D.W., Marotte, C., Verdier, R.I., et al. Comprehension during informed consent in a lessdeveloped country. Lancet. 2002; 360: 1301-1302.

14. Lidz, C.W. and Applebaum, P.S. The therapeutic misconception: Problems and solutions. Med. Care 2002; 40: Suppl: V55 - V 63.

15. Beauchamp, T.L. and Childress, J.F. Principles of Biomedical Ethics $4^{\text {th }}$ Ed. New York: Oxford Press, 1994.

16. Kirby, M.D. Informed consent: what does it mean? J. Med. Ethics. 1983; 9: 69-75.

17. McGrory, C.E., Friedland, B.A., Woodsong, C. and MacQueen, K.M. Informed consent in HIV prevention trials. Report of aninternational workshop. Population Council and Family Health International. 16-18, May 2005. New York.

18. CIOMS International guidelines for ethical review of epidemiological studies. In: Bankowski Z; BryantJ.H; Last J.M.eds., Ethics and Epidemiology: International Guidelines. Geneva: CIOMS, 1991; 167-190.

19. Fitzgerald, D.W., Wasunna, A. and Pape, J.W. Ten questions institutional review boards should ask when reviewing international clinical research protocols. IRB. 2003; 25: 14-18.

20. Kass, N., Dwason, L. and Loyo-Berrios, N.I. Ethical oversight of research in developing countries. IRB 2003; 25: 1-10.

21. Bereterbide, F. and Hirsch, F. Clinical trials in developing countries: who should define ethics? Bull. Soc. Pathol. Exot. 2008; 101: 102-105.

22. Lemmens, T. and Miller, P.B. The human subject trade: Ethical and legal issues surrounding recruitment incentives. J. Law Med. Ethics 2003; 31: 398-418.

23. Caulfield, T. and Griener, G. Conflict of interest in clinical research: addressing the issue of physician remuneration. J. Law Med. Ethics. 2002; 30: 305-308.

24. Molyneux, C.S., Peshu, N. and Marsh, K. Trust and informed consent; Insights from community members on the Kenyan coast. Soc. Sci. Med. 2005; 61: 1463-1473.
25. Barry M. Ethical considerations of human investigations in developing countries: the AIDS dilemma. New Engl. J. Med. 1988; 319: 1083-1085.

26. Moodley, K. HIV vaccine trial participation in South Africa - an ethical assessment. J. Med. Philos. 2002; 27: 197-215.

27. Bekelman, J.E. Li, Y. and Gross, C.P.Scope and impact of financial conflict of interest in biomedical research. J. Amer. Med. Assoc. 2003; 289: 462-463.

28. Lidz, C.W., Meisel, A., Osterweiss, M., et al. barriers to informed consent. Ann. Intern. Med. 1983; 99: 539-543.

29. Tobias, J.S. and Souhami, R.L. Fully informed consent can be needlessly cruel. Brit. Med. J. 1993; 307: 1199-1201.

30. Dickens, B.M. and Cook, R.J. Law and ethics in conflict over confidentiality? Int. J. Gynaecol. Obstet. 2000; 70: 385-391.

31. Henderson, G.E., Churchill, L.R., Davis, A.M., et al. Clinical trials and medical care: defining the therapeutic misconception. PLoS Med. 2007; 4:e324.doi:10.1371/ journal.pmed.0040324.

32. Benson, P.R., Roth, L.H. and Winslade, W.J. Informed consent in psychiatric research: preliminary findings from an ongoing investigation. Soc. Sci. Med. 1985; 20: 1331-1341.

33. Applebaum, P.S., Roth, L.H., Lidz, C.W., et al. False hopes and best data: consent to research and the therapeutic misconception. Hastings Cent. Rep. 1987; 17:20-24.

34. Schaeffer, M.H., Krantz, D.S., Wichman, A., Masur,H., et al. The impact of disease severity on the informed consent process in clinical research. Am. J. Med. 1996; 100: 261-268.

35. Roberts, L.W. Informed consent and the capacity for voluntarism. Am. J. Psychiatry. 2002; 159: 705-712.

36. Dickert, N. and Grady, C. What's the price of a research subject? Approaches to payment for research participation. N. Engl. J. Med. 1999; 341: 198-203.

37. Anderson, J. A. and Weijer, C. The research subject as wage earner. Theor. Med. Boieth. 2002; 23: 359-376.

38. Burgess, L., Sulzer, N. and Emanuel, S. Clinical trial remuneration: the patients' perspectives. South Afr. Med. J. 2008; 98: 95-97.

39. Moodley, K. and Myer, L. Participant remuneration for research - how much is enough? South Afr. Med. J. 2003; 93: 677-678.

40. Smit, J., Middlekoop, K., Myer, L., et al. Sociobehaviour challenges to phase III HIV vaccine trials in SSA. Afr. J. Health Sci. 2005; 5: 198-206.

41. Olivier, S. Informed consent and transcultural research. South. Afr. Med. J. 1995; 85: 984-985.

42. Leach, A. An evaluation of the informed consent procedure used during a trial of a Haemophilus influenzae type B conjugate vaccine undertaken in the Gambia, West Africa. Soc. Sci. Med. 1999; 48: 139-148,

43. Doyal, L. Informed consent - a response to recent correspondence. Brit. Med. J. 1998; 316: 1000-1001.

44. Montgomery, C., Lydon, A. and Lloyd, K. Patients may not understand enough to give their informed consent. Brit. Med. J. 1997; 314: 1482. 